\title{
RIGIDITY OF MORI CONE FOR FANO MANIFOLDS
}

\author{
JAROSŁAW A. WIŚNIEWSKI
}

Abstract. Mori cone is rigid in smooth connected families of Fano manifolds.

The aim of this note is to give a positive answer to a question raised at a workshop Rational curves on Algebraic Varieties organized in American Institute of Mathematics, Palo Alto, CA, in May 2007, Alt07, Question 0.7].

We consider algebraic varieties defined over the field of complex numbers $\mathbb{C}$. Manifolds are smooth connected varieties.

If $X$ is a complex projective manifold then by $N^{1}(X) \subset H^{2}(X, \mathbb{R})$ and $N_{1}(X) \subset H_{2}(X, \mathbb{R})$ we denote $\mathbb{R}$-linear subspaces spanned by cohomology and homology classes of, respectively, Cartier divisors and algebraic curves on $X$. The cone of curves, or Mori cone of $X, \mathcal{C}(X) \subset N_{1}(X)$ and the cone of nef divisors $\mathcal{P}(X) \subset N^{1}(X)$ are $\mathbb{R}_{>0}^{*}$-spanned by, respectively, the classes of curves, or effective 1-cycles, and numerically effective divisors, hence $\mathcal{P}(X):=\left\{\chi \in N^{1}(X): \forall \alpha \in\right.$ $\mathcal{C}(X) \quad \chi \cdot \alpha \geq 0\}$. That is, $\mathcal{P}(X)=\mathcal{C}(X)^{\vee}$ in the sense of the intersection pairing of $N^{1}(X)$ and $N_{1}(X)$. See [Mor82] for more information on these objects.

A manifold $X$ is Fano if its anticanonical divisor $-K_{X}$ is ample. If $X$ is Fano then $H^{1}\left(\mathcal{O}_{X}\right)=0$ hence $N^{1}(X)=H^{2}(X, \mathbb{R})$ and $N_{1}(X)=H_{2}(X, \mathbb{R})$.

A smooth family of projective manifolds $\pi: X \rightarrow S$ is, by definition, a smooth projective morphism of connected complex manifolds with connected fibers. Geometric fibers of $\pi$ are denoted by $X_{s}=\pi^{-1}(s)$ for $s \in S$. By a known result in differential geometry any such family is topologically locally trivial in complex topology. A local topological trivialization of $\pi$ induces identification of homology and cohomology of neighboring fibers.

Theorem 1. Let us assume that $\pi: X \rightarrow S$ is a smooth family of Fano manifolds, that is the relative anticanonical divisor $-K_{X / S}$ is $\pi$-ample. Then for any two $s_{0}, s_{1} \in S$ the local identification $H_{2}\left(X_{s_{0}}, \mathbb{R}\right)=H_{2}\left(X_{s_{1}}, \mathbb{R}\right)$ and $H^{2}\left(X_{s_{0}}, \mathbb{R}\right)=$ $H^{2}\left(X_{s_{1}}, \mathbb{R}\right)$ yields $\mathcal{C}\left(X_{s_{0}}\right)=\mathcal{C}\left(X_{s_{1}}\right)$ and $\mathcal{P}\left(X_{s_{0}}\right)=\mathcal{P}\left(X_{s_{1}}\right)$

The above result is an immediate consequence of the following main theorem from Wiś91, Theorem 1.7], see also Wiś98 where the ideas of [Wiś91, based on playing Hard Lefschetz Theorem against Mori's theory of rational curves, see Mor79, Mor82, were further developed. The notation in the present paper is consistent with that of Wiś91. Recall that given an ample line bundle $\mathcal{L}$ on a manifold $X$ its nef value, or nef threshold, $\tau(\mathcal{L})$ is the infimum of $t \in \mathbb{Q}$ such that $K_{X}+t \mathcal{L}$ is ample $\mathbb{Q}$-divisor. Note that $\tau(\mathcal{L})$ is positive if and only if $K_{X}$ is not nef.

Theorem 2. Let us assume that $\pi: X \rightarrow S$ is a smooth (connected) family of projective manifolds with $\mathcal{L}$ a $\pi$-ample line bundle. If $\tau\left(\mathcal{L}_{s_{0}}\right)$ is positive for some $s_{0} \in S$ then the function $S \ni s \mapsto \tau\left(\mathcal{L}_{s}\right)$ is constant.

1991 Mathematics Subject Classification. 14D06, 14E30, 14J45.

Research supported by a grant of Polish MNiSzW (N N201 2653 33). 
In view of Theorem 2 a proof of Theorem 1 can be reduced to choosing appropriate $\pi$-ample line bundle $\mathcal{L}$. Namely, suppose that $D_{0}$ is an ample divisor on $X_{s_{0}}$. Now, possibly shrinking $S$ to a connected base over which the family $\pi$ is topologically trivial we can extend $D_{0}$ to a divisor $\mathcal{D}$ over $X$; this extension provides us with an identification $H^{2}\left(X_{s_{0}}, \mathbb{R}\right)=H^{2}\left(X_{s_{1}}, \mathbb{R}\right)$. We claim that for a sufficiently small positive $\epsilon$ the divisor $\mathcal{L}=-K_{X}+\epsilon \cdot\left(\mathcal{D}+K_{X}\right)$ is $\pi$-ample. Indeed, since ampleness is an open condition, for any $s \in S$ there exists $\epsilon_{s}>0$ such that $-K_{X}+\epsilon_{s} \cdot\left(\mathcal{D}+K_{X}\right)$ is $\pi$-ample in an open Zariski neighborhood of $X_{s}$. Thus we can take $\epsilon=\min \left(\epsilon_{s_{0}}, \epsilon_{s_{1}}\right)$ and possibly shrink $S$ to a smaller connected variety containing both $s_{0}$ and $s_{1}$. We denote the resulting restricted family as before: $\pi: X \rightarrow S$. Now we apply Theorem 2 to argue that $\mathcal{D}_{s_{1}}$ is ample: see the picture below which presents a plane in $N^{1}\left(X_{s}\right)$ spanned on $K_{X}$ and $\mathcal{D}$; it is apparent that invariance of $\tau_{s}$ yields ampleness of $\mathcal{D}_{s}$ because the line determined by $K_{X}+\tau_{s} L_{s}$ is fixed and it constitues a border of the nef cone for all $s$. This implies that, in terms of the identification $H^{2}\left(X_{s_{0}}, \mathbb{R}\right)=H^{2}\left(X_{s_{1}}, \mathbb{R}\right)$, we get $\mathcal{P}\left(X_{s_{0}}\right)=\mathcal{P}\left(X_{s_{1}}\right)$. The equality $\mathcal{C}\left(X_{s_{0}}\right)=\mathcal{C}\left(X_{s_{1}}\right)$ follows by duality. This concludes a proof of Theorem 1

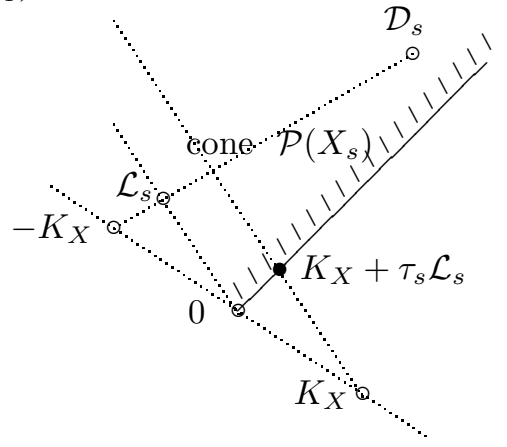

I would like to thank Rob Lazarsfeld who convinced me that it is worthwhile to write this note. He also observed that rigidity of the cone of big divisors for Fano manifolds follows from an extension theorem by Siu, [Siu02, and suggested the following corollary to Theorem 2

Theorem 3. Let $\pi: X \rightarrow S$ be a smooth family of projective manifolds. If for some $s_{0} \in S$ the variety $X_{s_{0}}$ is not minimal, that is $K_{s_{0}}$ is not nef, then no variety $X_{s}$ is minimal.

A version of the above theorem was proved in analytic category in AP97.

\section{REFERENCES}

[Alt07] Palo Alto. List of problems from the May 2007 American Institute of Mathematics Workshop on Rational Curves on Algebraic Varieties organized by B. Hasset and S. Kovács, http://www.aimath.org/pastworkshops/rationalcurves.pdf, 2007.

[AP97] M. Andreatta and T. Peternell. On the limits of manifolds with nef canonical bundles. In Complex analysis and geometry (Trento, 1995), volume 366 of Pitman Res. Notes Math. Ser., pages 1-6. Longman, Harlow, 1997.

[Mor79] Shigefumi Mori. Projective manifolds with ample tangent bundles. Ann. of Math. (2), 110(3):593-606, 1979.

[Mor82] Shigefumi Mori. Threefolds whose canonical bundles are not numerically effective. Ann. of Math. (2), 116(1):133-176, 1982.

[Siu02] Yum-Tong Siu. Extension of twisted pluricanonical sections with plurisubharmonic weight and invariance of semipositively twisted plurigenera for manifolds not necessarily of general type. In Complex geometry (Göttingen, 2000), pages 223-277. Springer, Berlin, 2002. 
[Wiś91] Jarosław A. Wiśniewski. On deformation of nef values. Duke Math. J., 64(2):325-332, 1991.

[Wiś98] Jarosław A. Wiśniewski. Cohomological invariants of complex manifolds coming from extremal rays. Asian J. Math., 2(2):289-301, 1998.

Instytut Matematyki UW, Banacha 2, PL-02097 Warszawa

E-mail address: J.Wisniewski@mimuw.edu.pl 